\title{
Treatment of Fulminant Hepatic Failure
}

\author{
Clinical Syndrome and Basis of Therapy
}

\author{
ROGER WILLIAMS
}

British Medical fournal, 1971, 1, 213-215

Fulminant hepatic failure has one of the highest mortalities among medical diseases- $82 \%$ in the first 318 patients reported from centres throughout the world to the Fulminant Hepatic Failure Surveillance (F.H.F.S.) study. ${ }^{1}$ The problem of fulminant hepatic failure is also being forcibly drawn to medical and public attention in this country by the continuing outbreaks of severe hepatitis in renal dialysis units. ${ }^{2}$

\section{Definition}

In the Boston study fulminant hepatic failure was defined as the clinical syndrome associated with massive necrosis of liver cells or with sudden severe impairment of hepatic function. ${ }^{1}$ It is characterized by an acute anset of progressive and severe mental changes starting with confusion and rapidly advancing to stupor or coma. Jaundice appears and rapidly increases in depth. Serum enzyme levels are markedly raised, and the prothrombin time considerably prolonged. Signs of encephalopathy usually appear within a few days of the onset, and the whole illness from first symptom to death may be less than a week. Less frequently patients are jaundiced for days or weeks before the appearance of neurological signs. The F.H.F.S. study therefore included within their definition all those patients in whom the signs described appear within eight weeks of the onset and in whom there had been no evidence of liver disease previously. Not included are those

King's College Hospital, London S.E.5

ROGER WILLIAMS, M.D., F.R.C.P., Director of Liver Unit and Consultant Physician patients with cirrhosis who develop signs of hepatic failure as a result of superimposed acute hepatic damage, as, for instance, following an alcoholic bout.

The cause in most cases is presumed to be viral hepatitis; other causes include direct toxic damage due to drugs (for instance, following an overdose of paracetamol) or from a hypersensitivity reaction (for instance, following halothane anaesthesia). Halothane-induced hepatic necrosis accounts for a substantial proportion of patients with fulminant hepatic failure-35 of the first 150 patients reported to the F.H.F.S. study. $^{3}$ The mortality is as high as $96 \% .^{4}$ Moreover, the disorder is largely preventable. An but one of the six patients seen at King's College Hospital over an 18-month period had had either unexplained fever or jaundice or both after the earlier anaesthetics. ${ }^{5}$

In fulminant hepatic failure there is usually widespread necrosis of hepatocytes with survival of bile ducts and ductules. The latter appear more numerous both because of collapse of the parenchyma and because of regeneration. In nearly all cases there is widespread inflammation in the portal tracts and in the lobules, to which I will return later.

\section{Prognostic Significance and Pathogenesis of Cerebral Manifestations}

Changes in mood, confusion, or drowsiness are usually the earliest detectable signs. The characteristic flapping tremor which is present in the early stages disappears as the patient sinks into coma. The survival rate of $66 \%$ in those with Grade II encephalopathy is to be compared with that of only $17.6 \%$ in those with Grade IV coma.

The very rapid onset of coma suggests a metabolic origin rather than structural changes, and in keeping with this is 
equally rapid reversal which can follow successful liver transplantation. Failure of the damaged liver to detoxicate substances absorbed into the blood following breakdown of nitrogenous substances in the colon is thought to be the main factor, but other metabolic changes as well as alterations in acid-base balance ${ }^{6}$ and possibly in cerebral blood flow may be additive. ${ }^{7}$ The primary site of the action is thought to be on the alerting mechanisms situated in the reticular formation.

When given orally the neurotransmitted amine L-dopa can produce a distinct improvement in conscious level and the electroencephalogram (E.E.G.) in hepatic coma. ${ }^{8}$ We do not yet know whether the alerting mechanisms in the reticular formation are mediated by dopaminergic reflexes or whether L-dopa will cause an arousal reaction in other metabolic comas.

\section{Principles of Therapy}

The basis of treatment in this condition is to support life long enough for spontaneous regeneration of the liver to occur. The liver has an extraordinary capacity for regeneration. In our and other series hepatic regeneration has occurred histologically in patients surviving over 10 days from the onset.

\section{Clinical Syndrome}

This may be considered under four main headings. The first of these is depression of brain stem function. In addition to drowsiness leading to coma, the respiratory and vasomotor centres fail. Sometimes this occurs early, and unless effective respiration and adequate circulation are maintained further anoxic cerebral damage is inevitable.

The second main set of changes are metabolic upsets and renal failure. Profound hypoglycaemia may occur and is often overlooked. ${ }^{9}$ This may produce flapping tremor and signs of disturbed pyramidal tract function and may be completely reversed by giving glucose. Hypokalaemia is present early; hyponatraemia tends to be a later development. Complicated changes in acid-base balance develop. The patient often hyperventilates initially, which may lead to respiratory alkalosis, and the hypokalaemia - as in other conditions-may be accompanied by a metabolic alkalosis. In addition, as a result of the extensive tissue necrosis, lactic acid and other metabolites can accumulate in the blood, thus leading to a metabolic acidosis. Renal failure with severe oliguria or anuria tends to develop particularly in the later stages and can be only partly explained by the circulatory hypotension.

Thirdly, there is failure of haemostasis (See next article).

Lastly, there is an increased incidence of infections. The unconscious state and inadequate ventilation favour respiratory infection, and the need for intravenous infusion and a urinary catheter predispose to infections by these routes. A Gram-negative septicaemia is a particular hazard.

\section{Basic Supportive Therapy}

The importance of intensive monitoring, including E.E.G. recordings, to detect complications at an early stage cannot be overemphasized. Withdrawal of oral protein, emptying of the bowel by an enema, and neomycin therapy to reduce bacterial growth in the colon are all important. With hepatic encephalopathy the brain becomes "oversensitive" to the effects of all sedative drugs, including chlorpromazine..$^{10}$ If necessary small doses of phenobarbitone, phenergan, or diazepam can be tried. The last is also useful for the minor convulsions that may occur.

The benefits of early tracheostomy must also be stressed. As a result of the central circulatory failure the circulation in these patients is extremely sensitive to volume depletion, for example gastrointestinal haemorrhage, and a central venous pressure line is essential in monitoring. If the central venous pressure is low without evidence of bleeding then the cautious infusion of salt-poor albumin may be beneficial.

The blood glucose should be measured four hourly. If hypoglycaemia does develop, massive amounts of glucose may be needed to correct this- $2.5 \mathrm{~kg}$ in 24 hours in the patient described by R. I. Samson and his colleagues. ${ }^{11}$ The serum amylase should be estimated daily, for several of these patients develop acute pancreatitis possibly from concomitant corticosteroid therapy.

The serum electrolytes also need frequent estimation. Supplementary potassium is nearly always needed, the amount depending on the changes in acid-base balance. A beneficial effect may result from the intravenous administration of 500 $\mathrm{ml}$ of $5 \%$ sodium bicarbonate solution. ${ }^{12}$ Renal failure, when it develops, is best treated by early rather than late peritoneal dialysis. One of the most difficult problems lies in preventing major bleeding, which by causing further hypotension has such deleterious effects on kidney and cerebral as well as on hepatic function. Finally, indwelling catheters should be changed every three days, and barrier nursing to minimize cross infection is also worthwhile.

In particularly fulminating cases it may be impossible to maintain life by supportive therapy alone, and some form of temporary liver support by extracorporeal pig liver perfusion or cross circulation will be necessary (see third article).

\section{Other and Possibly Specific Measures}

\section{CORTICOSTEROID THERAPY}

This is usually given and some benefit claimed, though there have been no controlled trials in fulminant hepatic failure. In man the possible beneficial effects have to be balanced against likely increases in the incidence of infections and of gastric erosions. Nevertheless, immune reactions may be important in the development of massive hepatic necrosis. J. D. Almeida and A. P. Waterson examined serum from one patient by electron microscopy and found immune complexes containing Australia antigen (the hepatitis-associated antigen), together with an excess of antibody. ${ }^{13}$ However, so far little else has been published on the incidence of hepatitis-associated antigen in this condition. Specific staining of the inflammatory infiltrate in the liver of 18 patients at necropsy showed that up to $80 \%$ of the mononuclear cells were pyroninophilic-indicating a high RNA content. ${ }^{16}$ Such cells are thought to be responsible for the tissue damage in transplanted organs undergoing rejection.

\section{EXCHANGE TRANSFUSION}

Of patients with fulminant hepatic failure $60 \%$ will temporarily regain consciousness following exchange transfusion, ${ }^{1}$ though the cause of this is not known. The recommended technique is to exchange one and a half times the calculated blood volume, the blood being infused rapidly into a vein and simultaneously withdrawn through an artery. Considerable care is needed to avoid swings in blood volume, and the blood pressure, pulse, and central venous pressure require careful monitoring. Furthermore, there is a certain risk to the staff performing the exchange.

Some of the initial enthusiasm for this procedure has waned, for the published long-term results are depressing. Of the 20 patients treated conservatively by S. J. Saunders and his colleagues from Cape Town, ${ }^{15}$ seven recovered consciousness and four survived; the respective figures for the 47 patients treated by exchange transfusion were 18 and 10 . However, these cases were not randomly allocated to the 
different treatments. Saunders carries out an exchange transfusion routinely if the patient remains in deep coma after 24 hours. In another personal series (from the Hospital Beaujon, Clichy) the results were quite different. ${ }^{16}$ Between 1952 and 196977 patients with severe viral hepatitis were seen, of whom 75 were in coma. Of 56 patients treated conservatively 13 survived, whereas there were no survivors in the 21 treated by corticosteroids, exchange transfusion, cross circulation, or hyperbaric oxygen. However, it is again quite likely that the more severe cases were treated by the more active measures. In the F.H.F.S. study of 101 patients in whom no treatment procedures were undertaken $10 \%$ survived, ${ }^{4}$ while $24 \%$ of 166 patients lived in whom exchange transfusions were done.

Thus no firm conclusions can be drawn about the value of exchange transfusion in the management of fulminant hepatic failure. Indeed the preliminary results in the first randomly allocated and controlled trial of exchange transfusion in fulminant hepatitis shows a higher mortality in the treated group. ${ }^{17}$

\section{References}

Trey, C., and Davidson, C. S., in Progress in Liver Diseases, ed. H. Popper and F, Schaffner, vol. 3. New York, Grune and Stratton, 1970.

British Medical fournal, 1970, 4, 255.

Trey, C., et al., New England fournal of Medicine, 1968, 279, 798.

' Trey, C., Lipworth, L., and Davidson, C. S., Gastroenterology, 1970, 58, 306 .

Sharpstone, P., Medley, D. R. K., and Williams, R., 1971, British Medical fournal. To be published.

Williams, R., and Toghill, P. J., Postgraduate Medical fournal, 1968, 44,

Polli, E., Porro, G. B., and Maiolo, A. T., Lancet, 1969, 1, 153.

Parkes, J. D., Sharpstone, P., and Williams, R., Lancet, 1970, 2, 1341.

Saunders, S. J., Bosman, S. C. W., Barnard, C. N., and Terblanche, J., Present Concepts, 1969, vol. 2. No. 7.

10 Read, A. E., Laidlaw, J., McCarthy, C. F., British Medical fournal, 1969, $3,497$.

Samson, R. I., Trey, C., Timme, A. H., and Saunders, S. J., Gastroenterology, 1967, 53, 291.

12 James, I. M., ot al., Lancet, 1969, 2, 1106.

13 Almeida, J. D., and Waterson, A. P., Lancet, 1969, 2, 983.

11 Goertz, G., Weinbren, H. K., and Williams, R., 1971, to be published.

Saunders, S. J., 1970. Personal communication Association for the Study of the Liver, Berne, 1970 .
Aster

Association for the Study of the Liver,
Redeker, A. G., personal communication.

\title{
Haemostasis in Fulminant Hepatic Failure
}

\author{
P. T. FLUTE
}

British Medical fournal, 1971, 1, 215-216

In fulminant hepatic failure the general reduction in protein synthesis by the liver leads to a fall in the plasma concentration of many clotting factors ${ }^{1}$, which is often sufficient to cause spontaneous bleeding. The concentration of clotting factors can sometimes be maintained, and bleeding prevented, by the transfusion of blood or blood products, but even this may be unsuccessful owing to an abnormally rapid loss of the transfused factors. Patients with hepatic coma have a fast clearance of fibrinogen, ${ }^{2}$ and indirect evidence suggests that platelets and coagulation factors other than fibrinogen are also involved in a process of intravascular coagulation.

\section{Intravascular Coagulation}

Many patients with fulminant hepatic failure show mild thrombocytopenia when platelet production is active. At the same time circulating fibrin degradation products are often increased. ${ }^{2}$ Hence the increased clearance of fibrinogen is probably due to consumption by coagulation in the blood stream. This "disseminated intravascular coagulation," "consumption coagulopathy," or "chronic defibrination," is seen in many states when blood comes into contact with damaged cells. ${ }^{3}$ In fulminant hepatic failure the stimulus probably comes from blood in contact with damaged liver cells.

Perhaps the most cogent argument in favour of intravascular coagulation, and the importance of its recognition, lies in the fact that heparin given to the patient tends to return fibrinogen clearance to normal. Such intravascular coagulation must throw an extra strain on the already overtaxed production capacity of the liver for clotting proteins. When their hepatic synthesis is severely reduced clotting factors can be

King's College Hospital Medical School, London S.E.5

P. T. FLUTE, M.D., M.R.C.PATH., Reader in Haematology increased only by transfusions of fresh or fresh frozen plasma, but their effect will last longer if heparin is given at the same time.

Probably all patients in hepatic coma may be assumed to have intravascular coagulation, though proof is difficult to obtain. The thrombin clotting time is prolonged when early breakdown products of fibrin or fibrinogen digestion are increased in the circulation, when the fibrinogen concentration is very low, or when circulating anticoagulants such as heparin are present. If the latter can be excluded, then a thrombin clotting time of $5 \mathrm{sec}$. or more longer than that of the control normal plasma is a typical finding of intravascular coagulation states.

\section{Causes of Bleeding}

The usual one-stage clotting tests-prothrombin time and partial thromboplastin time-do not indicate consumption directly but represent the balance achieved between production and loss of clotting factors. Together these simple tests cover the activity of almost the whole range of coagulation factors. The two tests are usually prolonged to a similar degree in hepatic coma but isolated readings are little guide to the risk of bleeding. Associated precipitating factors may have an important role-including damage to vessels (oesophageal varices, peptic ulcer, acute gastric erosions or surgical wounds) and a blood urea over $100 \mathrm{mg} / 100 \mathrm{ml}$. Bleeding, when it occurs, is often from the gastrointestinal tract, sometimes with skin purpura, and resembles the bleeding found in acute renal failure.

\section{Current Approach to Management}

Vitamin $\mathrm{K}$ is given to ensure that synthesis of clotting factors is not prevented by failure to absorb this vitamin. The onestage clotting tests are repeated daily. Patients with very 